\title{
Arene-Cations Interactions of Positive Quadrupole Moment Aromatics and Arene-Anion
}

Interactions of Negative Quadrupole Moment Interactions.

Aimee Clements and Michael Lewis*

Saint Louis University, Department of Chemistry, 3501 Laclede Avenue, Saint Louis, Missouri, USA 63103.

Table S1. RHF/6-311G**, MP2(full)/6-311G** (in italics), and MP2(full)/6-311++G** (in bold) Calculated Bond Distances and Bond Angles of $D_{2 h}$ Symmetric Aromatics. ${ }^{a}$

\begin{tabular}{|c|c|c|c|c|c|c|c|c|c|}
\hline $\mathrm{C}_{6} \mathrm{~F}_{4} \mathrm{I}_{2}$ & $\begin{array}{l}1.375 \\
1.391\end{array}$ & $\begin{array}{l}1.380 \\
1.395\end{array}$ & $\begin{array}{l}2.078 \\
2.060\end{array}$ & $\begin{array}{l}1.313 \\
1.334\end{array}$ & & $\begin{array}{l}121.2 \\
121.2\end{array}$ & $\begin{array}{l}117.5 \\
117.5\end{array}$ & $\begin{array}{l}121.2 \\
121.2\end{array}$ & $\begin{array}{l}120.7 \\
120.7\end{array}$ \\
\hline $\mathrm{C}_{6} \mathrm{H}_{2} \mathrm{~F}_{4}$ & $\begin{array}{l}1.376 \\
1.394 \\
\end{array}$ & $\begin{array}{l}1.376 \\
1.389 \\
\end{array}$ & $\begin{array}{l}1.073 \\
1.084 \\
\end{array}$ & $\begin{array}{l}1.317 \\
1.337 \\
\end{array}$ & & $\begin{array}{l}120.5 \\
120.3 \\
\end{array}$ & $\begin{array}{l}119.0 \\
119.4 \\
\end{array}$ & $\begin{array}{l}120.5 \\
120.3 \\
\end{array}$ & $\begin{array}{l}119.9 \\
120.0 \\
\end{array}$ \\
\hline $\mathrm{C}_{6} \mathrm{~F}_{4} \mathrm{Br}_{2}$ & $\begin{array}{l}1.376 \\
1.393 \\
\end{array}$ & $\begin{array}{l}1.380 \\
1.396 \\
\end{array}$ & $\begin{array}{l}1.875 \\
1.869 \\
\end{array}$ & $\begin{array}{l}1.309 \\
1.330 \\
\end{array}$ & & $\begin{array}{l}120.6 \\
120.5 \\
\end{array}$ & $\begin{array}{l}118.7 \\
119.0 \\
\end{array}$ & $\begin{array}{l}120.6 \\
120.5 \\
\end{array}$ & $\begin{array}{l}120.7 \\
120.7 \\
\end{array}$ \\
\hline $\mathrm{C}_{6} \mathrm{~F}_{4} \mathrm{Cl}_{2}$ & $\begin{array}{l}1.376 \\
1.392 \\
\end{array}$ & $\begin{array}{l}1.380 \\
1.396 \\
\end{array}$ & $\begin{array}{l}1.718 \\
1.712 \\
\end{array}$ & $\begin{array}{l}1.309 \\
1.330 \\
\end{array}$ & & $\begin{array}{l}120.6 \\
120.6 \\
\end{array}$ & $\begin{array}{l}118.8 \\
118.8 \\
\end{array}$ & $\begin{array}{l}120.6 \\
120.6 \\
\end{array}$ & $\begin{array}{l}120.5 \\
120.4 \\
\end{array}$ \\
\hline $\mathrm{C}_{6} \mathrm{H}_{2}(\mathrm{CN})_{4}$ & $\begin{array}{l}1.394 \\
1.414 \\
\end{array}$ & $\begin{array}{l}1.385 \\
1.400 \\
\end{array}$ & $\begin{array}{l}1.073 \\
1.085 \\
\end{array}$ & $\begin{array}{l}1.441 \\
1.431 \\
\end{array}$ & $\begin{array}{l}1.129 \\
1.177 \\
\end{array}$ & $\begin{array}{l}119.9 \\
119.9 \\
\end{array}$ & $\begin{array}{l}120.2 \\
120.2 \\
\end{array}$ & $\begin{array}{l}119.9 \\
119.9 \\
\end{array}$ & $\begin{array}{l}118.9 \\
119.8 \\
\end{array}$ \\
\hline $\mathrm{C}_{6} \mathrm{H}_{2} \mathrm{Cl}_{4}$ & $\begin{array}{l}1.385 \\
1.402 \\
\end{array}$ & $\begin{array}{l}1.381 \\
1.394 \\
\end{array}$ & $\begin{array}{l}1.072 \\
1.084\end{array}$ & $\begin{array}{l}1.730 \\
1.722 \\
\end{array}$ & & $\begin{array}{l}119.6 \\
119.4\end{array}$ & $\begin{array}{l}120.8 \\
121.0\end{array}$ & $\begin{array}{l}119.6 \\
119.4 \\
\end{array}$ & $\begin{array}{l}118.4 \\
118.8 \\
\end{array}$ \\
\hline $\mathrm{C}_{6} \mathrm{H}_{4} \mathrm{~F}_{2}$ & $\begin{array}{l}1.384 \\
1.396 \\
\mathbf{1 . 3 9 8} \\
\end{array}$ & $\begin{array}{l}1.377 \\
1.391 \\
\mathbf{1 . 3 9 1} \\
\end{array}$ & $\begin{array}{l}1.328 \\
1.346 \\
\mathbf{1 . 3 4 9} \\
\end{array}$ & $\begin{array}{l}1.074 \\
1.084 \\
\mathbf{1 . 0 8 4} \\
\end{array}$ & & $\begin{array}{l}119.0 \\
119.0 \\
\mathbf{1 1 8 . 9} \\
\end{array}$ & $\begin{array}{l}121.9 \\
121.9 \\
\mathbf{1 2 2 . 3} \\
\end{array}$ & $\begin{array}{l}119.0 \\
119.0 \\
\mathbf{1 1 8 . 9} \\
\end{array}$ & $\begin{array}{l}119.7 \\
119.6 \\
\mathbf{1 1 9 . 8} \\
\end{array}$ \\
\hline $\mathrm{C}_{6} \mathrm{H}_{4} \mathrm{Cl}_{2}$ & $\begin{array}{l}1.383 \\
1.395 \\
\mathbf{1 . 3 9 6} \\
\end{array}$ & $\begin{array}{l}1.381 \\
1.396 \\
\mathbf{1 . 3 9 7} \\
\end{array}$ & $\begin{array}{l}1.743 \\
1.733 \\
\mathbf{1 . 7 3 3} \\
\end{array}$ & $\begin{array}{l}1.074 \\
1.085 \\
\mathbf{1 . 0 8 5} \\
\end{array}$ & & $\begin{array}{l}119.5 \\
119.7 \\
\mathbf{1 1 9 . 7} \\
\end{array}$ & $\begin{array}{l}121.0 \\
120.6 \\
\mathbf{1 2 0 . 6} \\
\end{array}$ & $\begin{array}{l}119.5 \\
119.7 \\
\mathbf{1 1 9 . 7} \\
\end{array}$ & $\begin{array}{l}120.2 \\
119.9 \\
\mathbf{1 2 0 . 0} \\
\end{array}$ \\
\hline $\mathrm{C}_{6} \mathrm{H}_{2} \mathrm{I}_{4}$ & $\begin{array}{l}1.389 \\
1.405\end{array}$ & $\begin{array}{l}1.385 \\
1.398\end{array}$ & $\begin{array}{l}1.071 \\
1.085\end{array}$ & $\begin{array}{l}2.094 \\
2.075\end{array}$ & & $\begin{array}{l}118.9 \\
118.7\end{array}$ & $\begin{array}{l}122.2 \\
122.6\end{array}$ & $\begin{array}{l}118.9 \\
118.7\end{array}$ & $\begin{array}{l}116.7 \\
117.0\end{array}$ \\
\hline
\end{tabular}

\footnotetext{
${ }^{\mathrm{a}}$ Bond distances in $\AA$.
} 
Table S2. RHF/6-311G**, MP2(full)/6-311G** (in italics), and MP2(full)/6-311++G** (in bold) Calculated Bond Distances and Bond Angles of $D_{3 h}$ Symmetric Aromatics. ${ }^{a}$

\begin{tabular}{|c|c|c|c|c|c|c|c|c|}
\hline Aromatic & C-C & C-X & C-Y & C-N & $\mathrm{C}_{2}-\mathrm{C}_{1}-\mathrm{C}_{2}$ & $\mathrm{C}_{1}-\mathrm{C}_{2}-\mathrm{C}_{1}$ & $\mathrm{C}_{2}-\mathrm{C}_{1}-\mathrm{X}$ & $C_{1}-C_{2}-Y$ \\
\hline $\mathrm{C}_{6} \mathrm{H}_{3} \mathrm{~F}_{3}$ & $\begin{array}{l}1.378 \\
1.390\end{array}$ & $\begin{array}{l}1.072 \\
1.083\end{array}$ & $\begin{array}{l}1.319 \\
1.341\end{array}$ & & $\begin{array}{l}116.9 \\
117.2\end{array}$ & $\begin{array}{l}123.0 \\
122.8\end{array}$ & $\begin{array}{l}121.5 \\
121.4\end{array}$ & $\begin{array}{l}118.5 \\
118.6\end{array}$ \\
\hline $\mathrm{C}_{6} \mathrm{H}_{3}(\mathrm{CN})_{3}$ & $\begin{array}{l}1.387 \\
1.401\end{array}$ & $\begin{array}{l}1.073 \\
1.085\end{array}$ & $\begin{array}{l}1.442 \\
1.435 \\
\end{array}$ & $\begin{array}{l}1.129 \\
1.176\end{array}$ & $\begin{array}{l}119.5 \\
119.0\end{array}$ & $\begin{array}{l}120.5 \\
120.9\end{array}$ & $\begin{array}{l}120.0 \\
120.4\end{array}$ & $\begin{array}{l}119.8 \\
119.6 \\
\end{array}$ \\
\hline $\mathrm{C}_{6} \mathrm{H}_{3} \mathrm{Cl}_{3}$ & $\begin{array}{l}1.381 \\
1.395 \\
\mathbf{1 . 3 9 6}\end{array}$ & $\begin{array}{l}1.071 \\
1.083 \\
\mathbf{1 . 0 8 4}\end{array}$ & $\begin{array}{l}1.738 \\
1.730 \\
\mathbf{1 . 7 3 0}\end{array}$ & & $\begin{array}{l}118.2 \\
118.3 \\
\mathbf{1 1 8 . 3}\end{array}$ & $\begin{array}{l}121.8 \\
121.7 \\
\mathbf{1 2 1 . 7}\end{array}$ & $\begin{array}{l}120.9 \\
120.8 \\
\mathbf{1 2 0 . 8}\end{array}$ & $\begin{array}{l}119.0 \\
119.2 \\
\mathbf{1 1 9 . 2}\end{array}$ \\
\hline $\mathrm{C}_{6} \mathrm{H}_{3} \mathrm{I}_{3}$ & $\begin{array}{l}1.384 \\
1.398 \\
\mathbf{1 . 3 9 8}\end{array}$ & $\begin{array}{l}1.072 \\
1.085 \\
\mathbf{1 . 0 8 5}\end{array}$ & $\begin{array}{l}2.094 \\
2.078 \\
\mathbf{2 . 0 7 5}\end{array}$ & & $\begin{array}{l}119.5 \\
119.5 \\
\mathbf{1 1 9 . 7}\end{array}$ & $\begin{array}{l}120.5 \\
120.5 \\
\mathbf{1 2 0 . 3}\end{array}$ & $\begin{array}{l}120.3 \\
120.3 \\
\mathbf{1 2 0 . 1}\end{array}$ & $\begin{array}{l}119.7 \\
119.7 \\
\mathbf{1 1 9 . 9}\end{array}$ \\
\hline
\end{tabular}

${ }^{\mathrm{a}}$ Bond distances in $\AA$.

Table S3. RHF/6-311G**, MP2(full)/6-311G** (in italics), and MP2(full)/6-311++G** (in bold) Calculated Bond Distances and Bond Angles of $D_{6 h}$ Symmetric Aromatics. ${ }^{a}$

\begin{tabular}{|l|c|c|}
\hline \multicolumn{1}{|c|}{} & \multicolumn{2}{|c|}{} \\
\hline Aromatic & C-C & C-X \\
\hline $\mathrm{C}_{6} \mathrm{~F}_{6}$ & 1.377 & 1.309 \\
& 1.393 & 1.330 \\
& $\mathbf{1 . 3 9 3}$ & $\mathbf{1 . 3 3 0}$ \\
\hline $\mathrm{C}_{6} \mathrm{H}_{6}$ & 1.385 & 1.076 \\
& 1.398 & 1.086 \\
& $\mathbf{1 . 3 9 9}$ & $\mathbf{1 . 0 8 6}$ \\
\hline
\end{tabular}

\footnotetext{
${ }^{\mathrm{a}}$ Bond distances in $\AA$.
} 
Table S4. RHF/6-311G** and MP2(full)/6-311G** (in italics) Calculated Quadrupole Moment Tensor Components ( $\mathrm{Q}_{\mathrm{xx}}$, $\left.\mathrm{Q}_{\mathrm{yy}}, \mathrm{Q}_{\mathrm{zz}}\right)$ and Measurable Quadrupole Moments $\left(\Theta_{\mathrm{zz}}\right)$ of Substituted Aromatics. ${ }^{\mathrm{a}}$

\begin{tabular}{|c|c|c|c|c|}
\hline Aromatic & $\mathbf{Q}_{\mathrm{xx}}$ & $\mathbf{Q}_{\mathrm{yy}}$ & $\mathbf{Q}_{\mathrm{zz}}$ & $\Theta_{z z}$ \\
\hline \multirow[t]{2}{*}{$\mathrm{C}_{6} \mathrm{~F}_{4} \mathrm{I}_{2}$} & -120.09 & -100.44 & -109.76 & 0.51 \\
\hline & -121.14 & -100.08 & -109.98 & 0.63 \\
\hline \multirow[t]{2}{*}{$\mathrm{C}_{6} \mathrm{H}_{3} \mathrm{~F}_{3}$} & -50.97 & -50.97 & -50.28 & 0.69 \\
\hline & -51.44 & -51.44 & -50.55 & 0.90 \\
\hline \multirow{2}{*}{$\mathrm{C}_{6} \mathrm{~F}_{2} \mathrm{Cl}_{4}$} & -94.30 & -100.08 & -94.23 & 2.96 \\
\hline & -93.76 & -100.68 & -94.43 & 2.79 \\
\hline \multirow[t]{2}{*}{$\mathrm{C}_{6} \mathrm{H}_{2} \mathrm{~F}_{4}$} & -65.28 & -49.70 & -53.42 & 4.08 \\
\hline & -66.27 & -49.80 & -53.74 & 4.30 \\
\hline \multirow{2}{*}{$\mathrm{C}_{6} \mathrm{~F}_{4} \mathrm{Br}_{2}$} & -98.37 & -87.81 & -88.74 & 4.35 \\
\hline & -99.36 & -87.52 & -89.02 & 4.42 \\
\hline \multirow[t]{2}{*}{$\mathrm{C}_{6} \mathrm{~F}_{4} \mathrm{Cl}_{2}$} & -86.52 & -80.62 & -77.09 & 6.49 \\
\hline & -87.57 & -80.32 & -77.36 & 6.58 \\
\hline \multirow[t]{2}{*}{$\mathrm{C}_{6} \mathrm{~F}_{6}$} & -70.03 & -70.03 & -59.96 & 10.06 \\
\hline & -70.88 & -70.88 & -60.33 & 10.55 \\
\hline \multirow[t]{2}{*}{$\mathrm{C}_{6} \mathrm{H}_{4}(\mathrm{CN})_{2}$} & -47.06 & -94.75 & -59.09 & 11.81 \\
\hline & -47.21 & -95.73 & -59.70 & 11.78 \\
\hline \multirow[t]{2}{*}{$\mathrm{C}_{6} \mathrm{H}_{3}(\mathrm{CN})_{3}$} & -89.54 & -89.54 & -68.41 & 21.12 \\
\hline & -90.37 & -90.37 & -69.17 & 21.19 \\
\hline \multirow[t]{2}{*}{$\mathrm{C}_{6} \mathrm{H}_{2}(\mathrm{CN})_{4}$} & -124.80 & -86.54 & -77.83 & 27.84 \\
\hline & -126.60 & -86.10 & -78.79 & 27.56 \\
\hline \multirow[t]{2}{*}{$\mathrm{C}_{6} \mathrm{H}_{2} \mathrm{Cl}_{4}$} & -92.15 & -81.38 & -87.74 & -0.98 \\
\hline & -91.77 & -81.26 & -87.94 & -1.42 \\
\hline \multirow[t]{2}{*}{$\mathrm{C}_{6} \mathrm{H}_{3} \mathrm{Cl}_{3}$} & -74.18 & -74.18 & -75.95 & -1.76 \\
\hline & -73.94 & -73.94 & -76.17 & -2.23 \\
\hline \multirow[t]{2}{*}{$\mathrm{C}_{6} \mathrm{H}_{4} \mathrm{~F}_{2}$} & -36.69 & -52.76 & -46.90 & -2.17 \\
\hline & -36.50 & -53.42 & -47.19 & -2.23 \\
\hline \multirow[t]{2}{*}{$\mathrm{C}_{6} \mathrm{H}_{4} \mathrm{Cl}_{2}$} & -52.81 & -68.52 & -64.13 & -3.47 \\
\hline & -52.83 & -68.05 & -64.37 & -3.93 \\
\hline \multirow[t]{2}{*}{$\mathrm{C}_{6} \mathrm{H}_{2} \mathrm{Br}_{4}$} & -110.27 & -103.28 & -111.10 & -4.32 \\
\hline & -109.92 & -103.16 & -111.31 & -4.77 \\
\hline \multirow[t]{2}{*}{$\mathrm{C}_{6} \mathrm{H}_{4} \mathrm{Br}_{2}$} & -64.52 & -77.61 & -75.84 & -4.78 \\
\hline & -64.49 & -77.23 & -76.09 & -5.23 \\
\hline \multirow[t]{2}{*}{$\mathrm{C}_{6} \mathrm{H}_{4} \mathrm{I}_{2}$} & -86.10 & -92.36 & -96.96 & -7.73 \\
\hline & -86.11 & -91.89 & -97.17 & -8.17 \\
\hline \multirow[t]{2}{*}{$\mathrm{C}_{6} \mathrm{H}_{3} \mathrm{I}_{3}$} & -116.78 & -116.78 & -125.17 & -8.39 \\
\hline & -116.60 & -116.60 & -125.34 & -8.74 \\
\hline \multirow[t]{2}{*}{$\mathrm{C}_{6} \mathrm{H}_{6}$} & -31.68 & -31.68 & -40.43 & -8.76 \\
\hline & -31.64 & -31.64 & -40.71 & -9.07 \\
\hline \multirow[t]{2}{*}{$\mathrm{C}_{6} \mathrm{H}_{2} \mathrm{I}_{4}$} & -143.45 & -142.33 & -153.34 & -10.45 \\
\hline & -143.11 & -142.29 & -153.46 & -10.77 \\
\hline
\end{tabular}

${ }^{\text {a }}$ Quadrupole moment tensor components and measurable quadrupole moments in DÅ. All $\mathrm{C}_{6} \mathrm{X}_{4} \mathrm{Y}_{2}$ aromatics have $D_{2 h}$ symmetry, all $\mathrm{C}_{6} \mathrm{X}_{3} \mathrm{Y}_{3}$ aromatics have $D_{3 h}$ symmetry, and all $\mathrm{C}_{6} \mathrm{X}_{6}$ aromatics have $D_{6 h}$ symmetry. 
Table S5. RHF/6-311G**, MP2(full)/6-311G**, and MP2(full)/6-311++G** Calculated Total Energies, Thermal Energies at $298 \mathrm{~K}$ and the Number of Imaginary Frequencies (NI) of Aromatics, $\mathrm{Li}^{+}$and $\mathrm{F}^{-}$.

\begin{tabular}{|c|c|c|c|c|c|c|c|c|c|}
\hline \multirow{2}{*}{\begin{tabular}{|l|} 
Molecule \\
\end{tabular}} & \multicolumn{3}{|c|}{ RHF/6-311G** } & \multicolumn{3}{|c|}{ MP2(full)/6-311G** } & \multicolumn{3}{|c|}{ MP2(full)/6-311++G** } \\
\hline & Total Energy & \begin{tabular}{|c|} 
Thermal \\
Energy
\end{tabular} & NI & Total Energy & $\begin{array}{l}\text { Thermal } \\
\text { Energy }\end{array}$ & NI & Total Energy & $\begin{array}{l}\text { Thermal } \\
\text { Energy }\end{array}$ & NI \\
\hline $\mathrm{Li}^{+}$ & & & & -7.248321 & 0.89 & - & -7.248349 & 0.89 & - \\
\hline $\mathrm{Na}^{+}$ & & & & -161.793998 & 0.89 & & & & \\
\hline $\mathrm{C}_{6} \mathrm{~F}_{4} \mathrm{I}_{2}$ & -14399.905476 & 38.95 & 0 & -14401.961456 & 36.98 & 0 & & & \\
\hline $\mathrm{C}_{6} \mathrm{H}_{3} \mathrm{~F}_{3}$ & -527.386753 & 54.94 & 0 & -528.962212 & 52.01 & 0 & & & \\
\hline $\mathrm{C}_{6} \mathrm{~F}_{2} \mathrm{Cl}_{4}$ & -2264.155659 & 37.75 & 0 & -2266.271417 & 35.76 & 0 & & & \\
\hline $\mathrm{C}_{6} \mathrm{H}_{2} \mathrm{~F}_{4}$ & -626.245789 & 50.27 & 0 & -628.036735 & 47.48 & 0 & & & \\
\hline $\mathrm{C}_{6} \mathrm{~F}_{4} \mathrm{Br}_{2}$ & -5769.791222 & 38.88 & 0 & -5772.621239 & 36.65 & 0 & & & \\
\hline $\mathrm{C}_{6} \mathrm{~F}_{4} \mathrm{Cl}_{2}$ & -1544.065306 & 39.47 & 0 & -1546.231777 & 37.28 & 0 & & & \\
\hline $\mathrm{C}_{6} \mathrm{~F}_{6}$ & -823.966175 & 41.00 & 0 & -826.185864 & 38.53 & 0 & -826.227960 & 37.63 & 1 \\
\hline $\mathrm{C}_{6} \mathrm{H}_{4}(\mathrm{CN})_{2}$ & -414.255896 & 70.24 & 0 & -415.833976 & 65.34 & 0 & & & \\
\hline $\mathrm{C}_{6} \mathrm{H}_{3}(\mathrm{CN})_{3}$ & -505.999858 & 70.53 & 0 & -507.898128 & 65.31 & 0 & & & \\
\hline $\mathrm{C}_{6} \mathrm{H}_{2}(\mathrm{CN})_{4}$ & -597.732326 & 70.24 & 0 & -599.961430 & 64.64 & 0 & & & \\
\hline $\mathrm{F}^{-}$ & & & & -99.631990 & 0.89 & - & -99.697595 & 0.89 & - \\
\hline $\mathrm{Cl}^{-}$ & & & & -459.748839 & 0.89 & & & & \\
\hline $\mathrm{C}_{6} \mathrm{H}_{2} \mathrm{Cl}_{4}$ & -2066.423839 & 47.21 & 0 & -2068.107676 & 44.50 & 0 & & & \\
\hline $\mathrm{C}_{6} \mathrm{H}_{3} \mathrm{Cl}_{3}$ & -1607.514991 & 52.77 & 0 & -1609.008370 & 49.72 & 0 & -1609.026463 & 49.36 & 4 \\
\hline $\mathrm{C}_{6} \mathrm{H}_{4} \mathrm{~F}_{2}$ & -428.508530 & 59.78 & 0 & -429.871711 & 56.48 & 0 & -429.890191 & 56.13 & 0 \\
\hline $\mathrm{C}_{6} \mathrm{H}_{4} \mathrm{Cl}_{2}$ & -1148.596794 & 58.40 & 0 & -1149.903550 & 55.01 & 0 & -1149.917493 & 53.16 & 2 \\
\hline $\mathrm{C}_{6} \mathrm{H}_{2} \mathrm{Br}_{4}$ & -10517.868424 & 46.12 & 0 & -10520.881770 & 42.66 & 1 & -10520.896803 & 41.59 & 2 \\
\hline $\mathrm{C}_{6} \mathrm{H}_{4} \mathrm{Br}_{2}$ & -5374.319752 & 57.88 & 0 & -5376.290529 & 54.33 & 0 & -5376.301258 & 53.40 & 1 \\
\hline $\mathrm{C}_{6} \mathrm{H}_{4} \mathrm{I}_{2}$ & -14004.425691 & 58.04 & 0 & -14005.622496 & 54.65 & 0 & -14005.648214 & 53.42 & 1 \\
\hline $\mathrm{C}_{6} \mathrm{H}_{3} \mathrm{I}_{3}$ & -20891.259716 & 52.25 & 0 & -20892.588401 & 49.19 & 0 & -20892.623377 & 48.11 & 1 \\
\hline $\mathrm{C}_{6} \mathrm{H}_{6}$ & -230.754098 & 69.47 & 0 & -231.690871 & 65.39 & 0 & -231.697935 & 66.12 & 0 \\
\hline $\mathrm{C}_{6} \mathrm{H}_{2} \mathrm{I}_{4}$ & -27778.081748 & 46.29 & 0 & -27779.549282 & 43.75 & 0 & & & \\
\hline
\end{tabular}

${ }^{a}$ Total energies in hartrees and thermal energies in $\mathrm{kcal} / \mathrm{mol}$. All $\mathrm{C}_{6} \mathrm{X}_{4} \mathrm{Y}_{2}$ aromatics have $D_{2 h}$ symmetry, all $\mathrm{C}_{6} \mathrm{X}_{3} \mathrm{Y}_{3}$ aromatics have $D_{3 h}$ symmetry, and all $\mathrm{C}_{6} \mathrm{X}_{6}$ aromatics have $D_{6 h}$ symmetry. 


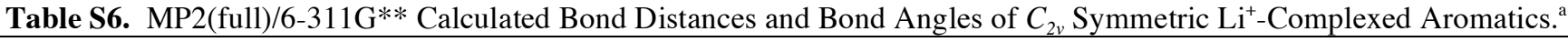

\begin{tabular}{|c|c|c|c|c|c|c|c|c|c|c|}
\hline & & & & & $\mathrm{Li}^{+}$ & & & & & \\
\hline $\begin{array}{c}\mathrm{Li}^{+} \\
\text {Complex }\end{array}$ & $\mathrm{C}_{2}-\mathrm{C}_{2}$ & $C_{1}-C_{2}$ & $\mathrm{C}-\mathrm{X}$ & $\mathrm{C}-\mathrm{Y}$ & $\mathbf{R}-\mathbf{L i}^{+}$ & C-N & $C_{1}-C_{2}-C_{2}$ & $C_{2}-C_{1}-C_{2}$ & $C_{2}-C_{1}-X$ & $\mathrm{C}_{1}-\mathrm{C}_{2}-\mathrm{Y}$ \\
\hline $\mathrm{C}_{6} \mathrm{~F}_{4} \mathrm{I}_{2}$ & 1.400 & 1.407 & 2.059 & 1.321 & 1.919 & & 121.4 & 117.0 & 121.4 & 120.5 \\
\hline $\mathrm{C}_{6} \mathrm{~F}_{2} \mathrm{Cl}_{4}$ & 1.415 & 1.407 & 1.318 & 1.700 & 1.932 & & 119.0 & 122.0 & 119.0 & 119.2 \\
\hline $\mathrm{C}_{6} \mathrm{H}_{2} \mathrm{~F}_{4}$ & 1.405 & 1.400 & 1.087 & 1.320 & 1.914 & & 120.0 & 120.0 & 120.0 & 120.3 \\
\hline $\mathrm{C}_{6} \mathrm{~F}_{4} \mathrm{Br}_{2}$ & 1.403 & 1.408 & 1.861 & 1.318 & 1.939 & & 120.7 & 118.5 & 120.7 & 120.5 \\
\hline $\mathrm{C}_{6} \mathrm{~F}_{4} \mathrm{Cl}_{2}$ & 1.402 & 1.408 & 1.700 & 1.317 & 1.939 & & 120.8 & 118.3 & 120.8 & 120.2 \\
\hline $\mathrm{C}_{6} \mathrm{H}_{4}(\mathrm{CN})_{2}$ & 1.400 & 1.413 & 1.432 & 1.087 & 1.942 & 1.177 & 120.0 & 119.9 & 120.0 & 119.6 \\
\hline $\mathrm{C}_{6} \mathrm{H}_{2}(\mathrm{CN})_{4}$ & 1.423 & 1.408 & 1.088 & 1.429 & 1.528 & 1.179 & 119.7 & 120.5 & 120.1 & 119.7 \\
\hline
\end{tabular}

${ }^{\mathrm{a}}$ Bond distances in $\AA$.

Table S7. MP2(full)/6-311G** Calculated Bond Distances and Bond Angles of $C_{3 v}$ Symmetric Li ${ }^{+}$-Complexed Aromatics. ${ }^{\text {a }}$

\begin{tabular}{|c|c|c|c|c|c|c|c|c|c|}
\hline $\begin{array}{c}\mathrm{Li}^{+} \\
\text {Complex }\end{array}$ & $\mathrm{C}-\mathrm{C}$ & $\mathrm{C}-\mathrm{X}$ & $\mathrm{C}-\mathrm{Y}$ & $\mathbf{R}-\mathbf{L i}^{+}$ & $\mathrm{C}-\mathrm{N}$ & $C_{2}-C_{1}-C_{2}$ & $C_{1}-C_{2}-C_{1}$ & $C_{2}-C_{1}-X$ & $C_{1}-C_{2}-Y$ \\
\hline $\mathrm{C}_{6} \mathrm{H}_{3}(\mathrm{CN})_{3}$ & 1.410 & 1.087 & 1.433 & 1.976 & 1.177 & 119.4 & 120.6 & 120.3 & 119.7 \\
\hline
\end{tabular}

${ }^{\mathrm{a}}$ Bond distances in $\AA$.

Table S8. MP2(full)/6-311G** and MP2(full)/6-311++G** (in bold) Calculated Bond Distances and Bond Angles of $C_{6 v}$ Symmetric $\mathrm{Li}^{+}$-Complexed Aromatics. ${ }^{a}$

\begin{tabular}{|c|c|c|c|}
\hline \multicolumn{4}{|c|}{$\mathrm{Li}^{+}$} \\
\hline $\mathrm{Li}^{+}$Complex & $\mathrm{C}-\mathrm{C}$ & C-X & R-Li ${ }^{+}$ \\
\hline $\mathrm{C}_{6} \mathrm{~F}_{6}$ & $\begin{array}{l}1.404 \\
\mathbf{1 . 4 0 4}\end{array}$ & $\begin{array}{l}1.316 \\
\mathbf{1 . 3 1 6}\end{array}$ & $\begin{array}{l}2.189 \\
\mathbf{2 . 1 4 8}\end{array}$ \\
\hline
\end{tabular}

\footnotetext{
${ }^{\mathrm{a}}$ Bond distances in $\AA$.
} 
Table S9. MP2(full)/6-311G** and MP2(full)/6-311++G** (in bold) Calculated Bond Distances and Bond Angles of $C_{2 v}$ Symmetric $\mathrm{F}^{-}$-Complexed Aromatics. ${ }^{\mathrm{a}}$

\begin{tabular}{|c|c|c|c|c|c|c|c|c|c|}
\hline $\begin{array}{c}\mathbf{F}^{-} \\
\text {Complex }\end{array}$ & $\mathrm{C}_{2}-\mathrm{C}_{2}$ & $\mathrm{C}_{1}-\mathrm{C}_{2}$ & C-X & C-Y & $\mathbf{R}-\mathbf{F}^{-}$ & $C_{1}-C_{2}-C_{2}$ & $C_{2}-C_{1}-C_{2}$ & $C_{2}-C_{1}-X$ & $\mathrm{C}_{1}-\mathrm{C}_{2}-\mathrm{Y}$ \\
\hline $\mathrm{C}_{6} \mathrm{H}_{2} \mathrm{Cl}_{4}$ & 1.393 & 1.387 & 1.082 & 1.731 & 2.470 & 119.8 & 120.5 & 119.7 & 118.5 \\
\hline $\mathrm{C}_{6} \mathrm{H}_{4} \mathrm{~F}_{2}$ & $\begin{array}{l}1.391 \\
\mathbf{1 . 3 9 5}\end{array}$ & $\begin{array}{l}1.384 \\
\mathbf{1 . 3 8 6}\end{array}$ & $\begin{array}{l}1.359 \\
\mathbf{1 . 3 6 1}\end{array}$ & $\begin{array}{l}1.084 \\
\mathbf{1 . 0 8 4}\end{array}$ & $\begin{array}{l}2.548 \\
\mathbf{2 . 8 2 5}\end{array}$ & $\begin{array}{l}118.7 \\
\mathbf{1 1 8 . 7}\end{array}$ & $\begin{array}{l}122.5 \\
\mathbf{1 2 2 . 6}\end{array}$ & $\begin{array}{l}118.8 \\
\mathbf{1 1 8 . 7}\end{array}$ & $\begin{array}{l}119.5 \\
\mathbf{1 1 9 . 8}\end{array}$ \\
\hline $\mathrm{C}_{6} \mathrm{H}_{4} \mathrm{Cl}_{2}$ & $\begin{array}{l}1.390 \\
1.393\end{array}$ & $\begin{array}{l}1.388 \\
\mathbf{1 . 3 9 1} \\
\end{array}$ & $\begin{array}{l}1.746 \\
\mathbf{1 . 7 4 3} \\
\end{array}$ & $\begin{array}{l}1.084 \\
\mathbf{1 . 0 8 5} \\
\end{array}$ & $\begin{array}{l}2.523 \\
\mathbf{2 . 7 6 8}\end{array}$ & $\begin{array}{l}119.2 \\
\mathbf{1 1 9 . 4}\end{array}$ & $\begin{array}{l}121.5 \\
\mathbf{1 2 1 . 2}\end{array}$ & $\begin{array}{l}119.2 \\
\mathbf{1 1 9 . 4}\end{array}$ & $\begin{array}{l}120.0 \\
\mathbf{1 2 0 . 1}\end{array}$ \\
\hline $\mathrm{C}_{6} \mathrm{H}_{2} \mathrm{Br}_{4}$ & $\begin{array}{l}1.392 \\
\mathbf{1 . 3 9 7}\end{array}$ & $\begin{array}{l}1.390 \\
\mathbf{1 . 3 9 3}\end{array}$ & $\begin{array}{l}1.082 \\
\mathbf{1 . 0 8 4}\end{array}$ & $\begin{array}{l}1.889 \\
\mathbf{1 . 8 8 7}\end{array}$ & $\begin{array}{l}2.452 \\
\mathbf{2 . 6 1 8}\end{array}$ & $\begin{array}{l}119.8 \\
\mathbf{1 1 9 . 8}\end{array}$ & $\begin{array}{l}120.4 \\
\mathbf{1 2 0 . 5}\end{array}$ & $\begin{array}{l}119.8 \\
\mathbf{1 1 9 . 7}\end{array}$ & $\begin{array}{l}117.6 \\
\mathbf{1 1 7 . 6}\end{array}$ \\
\hline $\mathrm{C}_{6} \mathrm{H}_{4} \mathrm{Br}_{2}$ & $\begin{array}{l}1.391 \\
1.394\end{array}$ & $\begin{array}{l}1.389 \\
\mathbf{1 . 3 9 2}\end{array}$ & $\begin{array}{l}1.903 \\
1.900\end{array}$ & $\begin{array}{l}1.084 \\
\mathbf{1 . 0 8 5}\end{array}$ & $\begin{array}{l}2.503 \\
\mathbf{2 . 7 5 2}\end{array}$ & $\begin{array}{l}119.0 \\
\mathbf{1 1 9 . 2}\end{array}$ & $\begin{array}{l}121.8 \\
\mathbf{1 2 1 . 5}\end{array}$ & $\begin{array}{l}119.0 \\
\mathbf{1 1 9 . 2}\end{array}$ & $\begin{array}{l}120.4 \\
\mathbf{1 2 0 . 5}\end{array}$ \\
\hline $\mathrm{C}_{6} \mathrm{H}_{4} \mathrm{I}_{2}$ & $\begin{array}{l}1.390 \\
1.392\end{array}$ & $\begin{array}{l}1.391 \\
1.393\end{array}$ & $\begin{array}{l}2.081 \\
\mathbf{2 . 0 7 6}\end{array}$ & $\begin{array}{l}1.085 \\
\mathbf{1 . 0 8 5}\end{array}$ & $\begin{array}{l}2.516 \\
\mathbf{2 . 7 8 5}\end{array}$ & $\begin{array}{l}119.9 \\
\mathbf{1 2 0 . 0}\end{array}$ & $\begin{array}{l}120.2 \\
\mathbf{1 2 0 . 0}\end{array}$ & $\begin{array}{l}119.9 \\
\mathbf{1 2 0 . 0}\end{array}$ & $\begin{array}{l}120.5 \\
\mathbf{1 2 0 . 5}\end{array}$ \\
\hline $\mathrm{C}_{6} \mathrm{H}_{2} \mathrm{I}_{4}$ & 1.397 & 1.391 & 1.083 & 2.077 & 2.450 & 119.0 & 122.0 & 119.0 & 116.7 \\
\hline
\end{tabular}

${ }^{\mathrm{a}}$ Bond distances in $\AA$.

Table S10. MP2(full)/6-311G** and MP2(full)/6-311++G** (in bold) Calculated Bond Distances and Bond Angles of $C_{3 v}$ Symmetric $\mathrm{F}^{-}$-Complexed Aromatics. ${ }^{\mathrm{a}}$

\begin{tabular}{|c|c|c|c|c|c|c|c|c|}
\hline $\begin{array}{c}\mathbf{F}^{-} \\
\text {Complex }\end{array}$ & $\mathrm{C}-\mathrm{C}$ & $\mathrm{C}-\mathrm{X}$ & C-Y & $\mathbf{R}-\mathbf{F}^{-}$ & $\mathbf{C}_{2}-\mathrm{C}_{1}-\mathrm{C}_{2}$ & $C_{1}-C_{2}-C_{1}$ & $\mathrm{C}_{2}-\mathrm{C}_{1}-\mathrm{X}$ & $\mathrm{C}_{1}-\mathrm{C}_{2}-\mathrm{Y}$ \\
\hline \multirow[t]{2}{*}{$\mathrm{C}_{6} \mathrm{H}_{3} \mathrm{Cl}_{3}$} & 1.388 & 1.083 & 1.743 & 2.483 & 117.5 & 122.5 & 121.2 & 118.7 \\
\hline & 1.405 & 1.080 & 1.770 & 2.748 & 120.7 & 119.3 & 120.4 & 119.6 \\
\hline $\mathrm{C}_{6} \mathrm{H}_{3} \mathrm{I}_{3}$ & $\begin{array}{l}1.390 \\
1.392\end{array}$ & $\begin{array}{l}1.083 \\
\mathbf{1 . 0 8 4}\end{array}$ & $\begin{array}{l}2.081 \\
\mathbf{2 . 0 7 6}\end{array}$ & $\begin{array}{l}2.476 \\
\mathbf{2 . 7 0 3}\end{array}$ & $\begin{array}{l}118.5 \\
\mathbf{1 2 1 . 2}\end{array}$ & $\begin{array}{l}121.5 \\
\mathbf{1 1 8 . 6}\end{array}$ & $\begin{array}{l}120.7 \\
\mathbf{1 2 0 . 5}\end{array}$ & $\begin{array}{l}119.3 \\
\mathbf{1 1 9 . 4}\end{array}$ \\
\hline
\end{tabular}

${ }^{\text {a }}$ Bond distances in $\AA$.

Table S11. MP2(full)/6-311G** and MP2(full)/6-311++G** (in bold) Calculated Bond Distances and Bond Angles of $C_{6 v}$ Symmetric $\mathrm{F}^{-}$-Complexed Aromatics. ${ }^{\mathrm{a}}$

\begin{tabular}{|c|c|c|c|}
\hline $\mathrm{F}^{-}$Complex & $\mathrm{C}-\mathrm{C}$ & C-X & $\mathbf{R}-\mathbf{F}^{-}$ \\
\hline $\mathrm{C}_{6} \mathrm{H}_{6}$ & $\begin{array}{l}1.392 \\
\mathbf{1 . 3 9 6}\end{array}$ & $\begin{array}{l}1.086 \\
\mathbf{1 . 0 8 7}\end{array}$ & $\begin{array}{l}2.786 \\
\mathbf{3 . 2 2 2}\end{array}$ \\
\hline
\end{tabular}

\footnotetext{
${ }^{\mathrm{a}}$ Bond distances in $\AA$.
} 
Table S12. MP2(full)/6-311G** Calculated Total Energies, Thermal Energies at $298 \mathrm{~K}$ and the Number of Imaginary Frequencies (NI) of $\mathrm{Li}^{+}-$and $\mathrm{F}^{-}$-Complexed Aromatics. ${ }^{\mathrm{a}}$

\begin{tabular}{|l|c|c|c|c|}
\hline Molecule & Energy & BSSE Corrected Energy & Thermal Energy & NI \\
\hline $\mathrm{C}_{6} \mathrm{~F}_{4} \mathrm{I}_{2}-\mathrm{Li}^{+}$ & -14409.248067 & -14409.221821 & 38.88 & 0 \\
\hline $\mathrm{C}_{6} \mathrm{H}_{3} \mathrm{~F}_{3}-\mathrm{Li}^{+}$ & -536.250530 & -536.242227 & 54.24 & 0 \\
\hline $\mathrm{C}_{6} \mathrm{~F}_{2} \mathrm{Cl}_{4}-\mathrm{Li}^{+}$ & -2273.552531 & -2273.539999 & 37.81 & 0 \\
\hline $\mathrm{C}_{6} \mathrm{H}_{2} \mathrm{~F}_{4}-\mathrm{Li}^{+}$ & -635.317846 & -635.308919 & 49.62 & 0 \\
\hline $\mathrm{C}_{6} \mathrm{~F}_{4} \mathrm{Br}_{2}-\mathrm{Li}^{+}$ & -5779.897698 & -5779.887531 & 38.62 & 0 \\
\hline $\mathrm{C}_{6} \mathrm{~F}_{4} \mathrm{Cl}_{2}-\mathrm{Li}^{+}$ & -1553.506215 & -1553.494992 & 39.31 & 0 \\
\hline $\mathrm{C}_{6} \mathrm{~F}_{6}-\mathrm{Li}^{+}$ & -833.453409 & -833.443046 & 40.61 & 0 \\
\hline $\mathrm{C}_{6} \mathrm{H}_{4}(\mathrm{CN})_{2}-\mathrm{Li}^{+}$ & -423.111961 & -423.104411 & 67.46 & 0 \\
\hline $\mathrm{C}_{6} \mathrm{H}_{3}(\mathrm{CN})_{3}-\mathrm{Li}^{+}$ & -515.161736 & -515.154452 & 67.10 & 0 \\
\hline $\mathrm{C}_{6} \mathrm{H}_{2}(\mathrm{CN})_{4}-\mathrm{Li}^{+}$ & -607.213081 & -607.205704 & 66.35 & 0 \\
\hline $\mathrm{C}_{6} \mathrm{H}_{2} \mathrm{Cl}_{4}-\mathrm{F}^{-}$ & -2167.776449 & -2167.746938 & 44.07 & 3 \\
\hline $\mathrm{C}_{6} \mathrm{H}_{3} \mathrm{Cl}_{3}-\mathrm{F}^{-}$ & -1708.671691 & -1708.642936 & 49.76 & 2 \\
\hline $\mathrm{C}_{6} \mathrm{H}_{4} \mathrm{~F}_{2}-\mathrm{F}^{-}$ & -529.524551 & -529.498107 & 56.44 & 2 \\
\hline $\mathrm{C}_{6} \mathrm{H}_{4} \mathrm{Cl}_{2}-\mathrm{F}^{-}$ & -1249.559219 & -1249.531743 & 54.88 & 2 \\
\hline $\mathrm{C}_{6} \mathrm{H}_{2} \mathrm{Br}_{4}-\mathrm{F}^{-}$ & -10620.550442 & -10620.520792 & 42.91 & 3 \\
\hline $\mathrm{C}_{6} \mathrm{H}_{4} \mathrm{Br}_{2}-\mathrm{F}^{-}$ & -5475.946785 & -5475.919226 & 53.66 & 3 \\
\hline $\mathrm{C}_{6} \mathrm{H}_{4} \mathrm{I}_{2}-\mathrm{F}^{-}$ & -14105.275909 & -14105.280841 & 54.54 & 2 \\
\hline $\mathrm{C}_{6} \mathrm{H}_{3} \mathrm{I}_{3}-\mathrm{F}^{-}$ & -20992.247837 & -20992.248074 & 49.23 & 2 \\
\hline $\mathrm{C}_{6} \mathrm{H}_{6}-\mathrm{F}^{-}$ & -331.328347 & -331.304962 & 64.21 & 3 \\
\hline $\mathrm{C}_{6} \mathrm{H}_{2} \mathrm{I}_{4}-\mathrm{F}^{-}$ & -27879.212261 & -27879.209755 & 43.96 & 2 \\
\hline
\end{tabular}

${ }^{a}$ Total energies in hartrees and thermal energies in kcal/mol. All $\mathrm{C}_{6} \mathrm{X}_{4} \mathrm{Y}_{2}-\mathrm{Li}^{+}$and $\mathrm{C}_{6} \mathrm{X}_{4} \mathrm{Y}_{2}-\mathrm{F}^{-}$complexes have $C_{2 v}$ symmetry, all $\mathrm{C}_{6} \mathrm{X}_{3} \mathrm{Y}_{3}-\mathrm{Li}^{+}$and $\mathrm{C}_{6} \mathrm{X}_{3} \mathrm{Y}_{3}-\mathrm{F}^{-}$complexes have $C_{3 v}$ symmetry, and all $\mathrm{C}_{6} \mathrm{X}_{6}-\mathrm{Li}^{+}$and $\mathrm{C}_{6} \mathrm{X}_{6}-\mathrm{F}^{-}$complexes have $C_{6 v}$ symmetry.

Table S13. MP2(full)/6-311G** Calculated Bond Distances and Bond Angles of $C_{2 v}$ Symmetric $\mathrm{Na}^{+}$-Complexed Aromatics. ${ }^{\mathrm{a}}$

\begin{tabular}{|c|c|c|c|c|c|c|c|c|c|c|}
\hline \multicolumn{11}{|c|}{$\mathrm{Na}^{+}$} \\
\hline $\begin{array}{c}\mathrm{Na}^{+} \\
\text {Complex }\end{array}$ & $\mathrm{C}_{2}-\mathrm{C}_{2}$ & $\mathrm{C}_{1}-\mathrm{C}_{2}$ & C-X & $\mathrm{C}-\mathrm{Y}$ & $\mathbf{R}-\mathrm{Na}^{+}$ & C-N & $\mathrm{C}_{1}-\mathrm{C}_{2}-\mathrm{C}_{2}$ & $\mathrm{C}_{2}-\mathrm{C}_{1}-\mathrm{C}_{2}$ & $\mathrm{C}_{2}-\mathrm{C}_{1}-\mathrm{X}$ & $C_{1}-C_{2}-Y$ \\
\hline $\mathrm{C}_{6} \mathrm{~F}_{4} \mathrm{I}_{2}$ & 1.399 & 1.404 & 2.063 & 1.327 & 2.476 & & 121.4 & 117.1 & 121.4 & 120.6 \\
\hline $\mathrm{C}_{6} \mathrm{~F}_{2} \mathrm{Cl}_{4}$ & 1.412 & 1.404 & 1.324 & 1.706 & 2.490 & & 119.0 & 122.0 & 119.0 & 119.1 \\
\hline $\mathrm{C}_{6} \mathrm{H}_{2} \mathrm{~F}_{4}$ & 1.402 & 1.397 & 1.087 & 1.327 & 2.465 & & 120.1 & 119.7 & 120.1 & 120.3 \\
\hline $\mathrm{C}_{6} \mathrm{~F}_{4} \mathrm{Br}_{2}$ & 1.400 & 1.405 & 1.865 & 1.324 & 2.518 & & 120.7 & 118.6 & 120.5 & 120.7 \\
\hline $\mathrm{C}_{6} \mathrm{~F}_{4} \mathrm{Cl}_{2}$ & 1.399 & 1.405 & 1.705 & 1.323 & 2.517 & & 120.8 & 118.4 & 120.7 & 120.3 \\
\hline $\mathrm{C}_{6} \mathrm{H}_{4}(\mathrm{CN})_{2}$ & 1.399 & 1.412 & 1.434 & 1.087 & 2.520 & 1.178 & 120.0 & 120.0 & 120.0 & 119.6 \\
\hline $\mathrm{C}_{6} \mathrm{H}_{2}(\mathrm{CN})_{4}$ & 1.421 & 1.406 & 1.086 & 1.431 & 2.270 & 1.179 & 119.8 & 120.4 & 119.8 & 120.0 \\
\hline
\end{tabular}

${ }^{\mathrm{a}}$ Bond distances in $\AA$. 
Table S14. MP2(full)/6-311G** Calculated Bond Distances and Bond Angles of $C_{3 v}$ Symmetric $\mathrm{Na}^{+}$-Complexed Aromatics. ${ }^{a}$

\begin{tabular}{|c|c|c|c|c|c|c|c|c|c|}
\hline \multicolumn{10}{|c|}{$\mathrm{Na}^{+}$} \\
\hline $\begin{array}{c}\mathrm{Na}^{+} \\
\text {Complex }\end{array}$ & C-C & C-X & $\mathrm{C}-\mathrm{Y}$ & $\mathbf{R}-\mathrm{Na}^{+}$ & C-N & $\mathrm{C}_{2}-\mathrm{C}_{1}-\mathrm{C}_{2}$ & $\mathrm{C}_{1}-\mathrm{C}_{2}-\mathrm{C}_{1}$ & $\mathrm{C}_{2}-\mathrm{C}_{1}-\mathrm{X}$ & $C_{1}-C_{2}-Y$ \\
\hline $\mathrm{C}_{6} \mathrm{H}_{3} \mathrm{~F}_{3}$ & 1.398 & 1.086 & 1.329 & 2.472 & & 117.4 & 122.5 & 121.3 & 118.7 \\
\hline $\mathrm{C}_{6} \mathrm{H}_{3}(\mathrm{CN})_{3}$ & 1.408 & 1.088 & 1.435 & 2.588 & 1.178 & 119.3 & 120.7 & 120.3 & 119.7 \\
\hline
\end{tabular}

${ }^{\mathrm{a}}$ Bond distances in $\AA$.

Table S15. MP2(full)/6-311G** Calculated Bond Distances and Bond Angles of $C_{6 v}$ Symmetric $\mathrm{Na}^{+}$-Complexed Aromatics. ${ }^{\mathrm{a}}$

\begin{tabular}{|c|c|c|c|}
\hline \multicolumn{4}{|c|}{$\mathrm{Na}^{+}$} \\
\hline $\mathrm{Na}^{+}$Complex & C-C & C-X & $\mathbf{R}-\mathbf{N a}^{+}$ \\
\hline $\mathrm{C}_{6} \mathrm{~F}_{6}$ & 1.401 & 1.322 & 2.148 \\
\hline
\end{tabular}

${ }^{\mathrm{a}}$ Bond distances in $\AA$.

Table S16. MP2(full)/6-311G** Calculated Total Energies, Thermal Energies at $298 \mathrm{~K}$ and the Number of Imaginary Frequencies (NI) of $\mathrm{Na}^{+}$-Complexed Aromatics. ${ }^{a}$

\begin{tabular}{|l|c|c|c|c|}
\hline Molecule & Energy & BSSE Corrected Energy & Thermal Energy & NI \\
\hline $\mathrm{C}_{6} \mathrm{~F}_{4} \mathrm{I}_{2}-\mathrm{Na}^{+}$ & -161.793998 & -14563.765242 & 38.66 & 0 \\
\hline $\mathrm{C}_{6} \mathrm{H}_{3} \mathrm{~F}_{3}-\mathrm{Na}^{+}$ & -14563.775774 & -536.235485 & 53.94 & 0 \\
\hline $\mathrm{C}_{6} \mathrm{~F}_{2} \mathrm{Cl}_{4}-\mathrm{Na}^{+}$ & -690.777665 & -2428.071734 & 37.53 & 0 \\
\hline $\mathrm{C}_{6} \mathrm{H}_{2} \mathrm{~F}_{4}-\mathrm{Na}^{+}$ & -2428.081029 & -789.839782 & 49.34 & 0 \\
\hline $\mathrm{C}_{6} \mathrm{~F}_{4} \mathrm{Br}_{2}-\mathrm{Na}^{+}$ & -789.846558 & -5934.420026 & 38.37 & 0 \\
\hline $\mathrm{C}_{6} \mathrm{~F}_{4} \mathrm{Cl}_{2}-\mathrm{Na}^{+}$ & -5934.427490 & -1708.028025 & 39.04 & 0 \\
\hline $\mathrm{C}_{6} \mathrm{~F}_{6}-\mathrm{Na}^{+}$ & -1708.036167 & -987.977619 & 40.32 & 0 \\
\hline $\mathrm{C}_{6} \mathrm{H}_{4}(\mathrm{CN})_{2}-\mathrm{Na}^{+}$ & -987.985161 & -577.635324 & 67.12 & 0 \\
\hline $\mathrm{C}_{6} \mathrm{H}_{3}(\mathrm{CN})_{3}-\mathrm{Na}^{+}$ & -577.641085 & -669.688730 & 66.85 & 0 \\
\hline $\mathrm{C}_{6} \mathrm{H}_{2}(\mathrm{CN})_{4}-\mathrm{Na}^{+}$ & -669.693973 & -761.743337 & 66.07 & 0 \\
\hline
\end{tabular}

${ }^{\mathrm{a}}$ Total energies in hartrees and thermal energies in kcal/mol. All $\mathrm{C}_{6} \mathrm{X}_{4} \mathrm{Y}_{2}-\mathrm{Na}^{+}$complexes have $C_{2 v}$ symmetry, all $\mathrm{C}_{6} \mathrm{X}_{3} \mathrm{Y}_{3}-\mathrm{Na}^{+}$ complexes have $C_{3 v}$ symmetry, and all $\mathrm{C}_{6} \mathrm{X}_{6}-\mathrm{Na}^{+}$complexes have $C_{6 v}$ symmetry. 
Table S17. MP2(full)/6-311++G** Calculated Total Energies, Thermal Energies at $298 \mathrm{~K}$ and the Number of Imaginary Frequencies (NI) of $\mathrm{Li}^{+}-$and $\mathrm{F}^{-}$-Complexed Aromatics. ${ }^{\mathrm{a}}$

\begin{tabular}{|l|c|c|c|c|}
\hline Molecule & Energy & BSSE Corrected Energy & Thermal Energy & NI \\
\hline $\mathrm{C}_{6} \mathrm{~F}_{6}-\mathrm{Li}^{+}$ & -833.489339 & -833.482994 & 39.68 & 1 \\
\hline $\mathrm{C}_{6} \mathrm{H}_{3} \mathrm{Cl}_{3}-\mathrm{F}$ & -1708.742459 & -1708.730062 & 49.36 & 7 \\
\hline $\mathrm{C}_{6} \mathrm{H}_{4} \mathrm{~F}_{2}-\mathrm{F}$ & -529.597438 & -529.594620 & 56.36 & 2 \\
\hline $\mathrm{C}_{6} \mathrm{H}_{4} \mathrm{Cl}-\mathrm{F}$ & -1249.626787 & -1249.622776 & 54.30 & 3 \\
\hline $\mathrm{C}_{6} \mathrm{H}_{2} \mathrm{Br}_{4}-\mathrm{F}$ & -10620.617626 & -10620.610335 & 42.24 & 4 \\
\hline $\mathrm{C}_{6} \mathrm{H}_{4} \mathrm{Br}_{2}-\mathrm{F}$ & -5476.011259 & -5476.007455 & 53.89 & 3 \\
\hline $\mathrm{C}_{6} \mathrm{H}_{4} \mathrm{I}_{2}-\mathrm{F}$ & -14105.357703 & -14105.352515 & 53.52 & 4 \\
\hline $\mathrm{C}_{6} \mathrm{H}_{3} \mathrm{I}_{3}-\mathrm{F}$ & -20992.339137 & -20992.329700 & 49.53 & 3 \\
\hline $\mathrm{C}_{6} \mathrm{H}_{6}-\mathrm{F}$ & -331.393366 & -331.391455 & 64.31 & 3 \\
\hline
\end{tabular}

${ }^{\mathrm{a}}$ Total energies in hartrees and thermal energies in $\mathrm{kcal} / \mathrm{mol}$. All $\mathrm{C}_{6} \mathrm{X}_{4} \mathrm{Y}_{2}-\mathrm{F}^{-}$complexes have $C_{2 v}$ symmetry and all $\mathrm{C}_{6} \mathrm{X}_{6}-\mathrm{Li}^{+}$ and $\mathrm{C}_{6} \mathrm{X}_{6}-\mathrm{F}^{-}$complexes have $C_{6 v}$ symmetry. 